\title{
Option
}

\section{La biodiversité : \\ un nouveau regard sur la diversité du vivant *}

\author{
II. Stabilité et utilité
}

\section{Bernard Chevassus-au-Louis}

Institut national de la recherche agronomique (Inra),

Laboratoire de génétique des poissons,

Domaine de Vilvert,

78350 Jouy-en-Josas

<bernard.chevassus@jouy.inra.fr>

\begin{abstract}
Résumé
L'introduction et le succès du terme "biodiversité " ont concrétisé plusieurs évolutions récentes dans notre perception et notre compréhension de la diversité des êtres vivants. Nous avons évoqué dans une première partie deux aspects de ces évolutions, la prise de conscience de l'ampleur insoupçonnée de la diversité spécifique, d'une part, et, d'autre part, la nécessité de prendre en compte d'autres niveaux d'organisation que celui de la diversité des espèces, en particulier la diversité individuelle intra-spécifique et, au niveau supraspécifique, la diversité des biocénoses et des écosystèmes. Nous examinons ici deux autres aspects de cette nouvelle vision de la diversité du vivant : i) un changement de perspective vis-à-vis de l'utilité de la biodiversité. Auparavant souvent considérée à l'aune de la fourniture de biens marchands (aliments, matériaux, médicaments), cette notion d'utilité s'ouvre peu à peu à d'autres composantes jouant, ou susceptibles de jouer à l'avenir, un rôle beaucoup plus crucial: innovations tirées de l'imitation du vivant, services écologiques, rôle de sentinelle vis-à-vis des polluants ; ii) la remise en cause de la notion de stabilité. Conçue préalablement de manière statique - et donnant lieu de ce fait à des mesures de conservation rigide -, cette stabilité doit désormais être perçue comme un équilibre dynamique, dans lequel certaines perturbations peuvent jouer un rôle bénéfique. De ce fait, les stratégies de conservation doivent désormais veiller à préserver les capacités d'évolution beaucoup plus qu'un état à un instant donné.
\end{abstract}

Mots clés : biodiversité ; durabilité ; utilité.

Thèmes : ressources naturelles et environnement.

\section{Abstract \\ Biodiversity: a new look at the diversity of life. II. Stability and usefulness}

The successful emergence of the term "biodiversity" expresses several recent changes in our perception and understanding of the diversity of living organisms. In an initial article we evoked two aspects of these changes, firstly the sudden awareness of the unexpected magnitude of the diversity of species and, secondly, the need to consider the existence, in addition to the diversity of species, of other levels of organization, mainly individualwithin-species diversity and, at the supraspecific level, the diversity of biocenosis and ecosystems. In this article, we present two other aspects characterizing this new vision of the diversity of life: i) a new way of considering the usefulness of biodiversity. Instead of being focused on the production of marketable goods (foods, drugs, raw materials), usefulness now takes into account other aspects that can play a more crucial role in the future: source of innovations based on the mimicry of living organisms (biomimesis), production of ecological services, early and integrative detection of pollutants; ii) a reconsideration of the concept of stability. Instead of being conceived in a static way, with conservation policies based on rigid measures, stability is now perceived as a dynamic

* Ce texte est une version amendée et actualisée d'une conférence présentée le 16 décembre 2004 à l'Académie vétérinaire de France sous le titre : Les enjeux de la biodiversité animale. (Bull Acad Vet France 2005 ; 158 : 91-110). 
equilibrium in which some perturbations can have favourable influences. As a result, conservation strategies now have to protect the evolutionary capabilities of biodiversity much better than simply at a particular state or time.

Key words: biodiversity; sustainability; usefulness.

Subjects: natural resources and environment.

e terme "biodiversité " symbolise l'émergence et la convergence de nouvelles visions de cette diversité du vivant, aboutissant globalement à proposer un "nouveau regard" qui met en lumière cinq aspects principaux de cette diversité :

- son immensité, insoupçonnée jusqu'alors ;

- sa complexité, liée à ses différents niveaux d'organisation et à leurs interactions ;

- sa stabilité, qui ne doit pas être conçue comme un équilibre statique mais s'inscrit dans une dynamique permanente ;

- son utilité, à travers l'ensemble des " services écologiques " qu'elle apporte ;

- sa fragilité enfin, avec la prise de conscience des impacts humains, souvent irréversibles, sur des ressources que l'on croyait, parce que vivantes, inépuisables. Après avoir exploré les deux premières notions dans un précédent article (Chevassus-au-Louis, 2007), nous examinons ici les aspects relatifs à la stabilité et à l'utilité.

\section{L'utilité : des fonctions méconnues}

Face au défi d'inventorier et, surtout, de préserver un ensemble dont l'ampleur et la complexité apparaissent aujourd'hui "inestimables" au cours du présent siècle, la question de l'utilité de la biodiversité est souvent posée. Nous utilisons ici le terme "utilité "dans son sens le plus courant d'utilisation possible d'une entité naturelle ou créée par l'homme (à quoi cela peut-il servir?) et non au sens plus technique que l'on rencontre en particulier en économie et qui implique un marché (au moins fictif) et des "acquéreurs " potentiels de cette entité. Nous nous limiterons à un point de vue anthropocentrique, en examinant essentiellement la question de l'utilité pour l'espèce humaine.

Nous présenterons tout d'abord les arguments les plus fréquemment échangés, pour nous attacher ensuite à montrer que les aspects les plus importants ne sont peut-être pas ceux qui retiennent immédiatement l'attention.

\section{Les arguments " classiques "}

Confrontés à cette question de l'utilité, les défenseurs de la biodiversité développent le plus souvent deux types d'argumentaires.

Le premier consiste à dénoncer cette vision "utilitariste" et à souligner que d'autres raisons, éthiques, esthétiques, culturelles, suffisent à justifier leur attitude. Ainsi, l'introduction du concept de "patrimoine naturel" permet de relier explicitement la protection de la nature à celle des œuvres de l'homme - tableaux, musiques, monuments - qui, on en conviendra, n'est pas fondée sur une vision strictement utilitariste. De même, la référence à d'autres cadres éthiques ${ }^{1}$ qu'un anthropocentrisme à court terme permet de retourner le questionnement et de s'interroger sur les fondements du droit que l'homme s'attribue de perturber la nature et de faire disparaître, volontairement ou involontairement, des espèces. Complétant la dénonciation des visions discriminatoires $\mathrm{du}$ sexisme et $\mathrm{du}$ racisme, la dénonciation du "spécisme ", à savoir de la conception de la supériorité de notre espèce vis-à-vis des autres espèces vivantes, est l'exemple emblématique de cette remise en cause (Porcher, 2002). Le second registre consiste, à l'inverse, à rentrer dans cette logique d'utilitarisme immédiat et à montrer tout ce que la connaissance et l'utilisation de la biodiversité ont permis à l'homme de faire

\footnotetext{
$\overline{1}$ Nous renvoyons à notre article Chevassusau-Louis et al. (2004) pour une présentation de ces différentes éthiques de la protection de la nature.
}

depuis ses origines. Le fait que l'agriculture et l'alimentation européennes soient en grande partie fondées sur des espèces collectées dans l'ensemble de la planète est connu mais constitue dans ce contexte un argument fort: les trois symboles de l'identité française - le pain, le vin et le fromage $^{2}$ - sont issus d'espèces qui n'étaient pas présentes sur notre territoire au néolithique; de même, plusieurs emblèmes de l'alimentation méditerranéenne - tomates, poivrons, aubergines n'ont rejoint qu'assez récemment cette région. On peut aussi faire remarquer que les bateaux qui ont permis d'explorer le monde et d'en rapporter toutes ces espèces sont eux-mêmes issus d'une utilisation pertinente de la biodiversité : diversité des essences d'arbres pour la coque (en chêne), les mâts (en sapin), les poulies (en buis), les avirons (en hêtre), les affûts de canon (en orme); diversité des fibres textiles pour les cordages, les voiles, le calfatage, etc.

Enfin, l'utilisation par l'homme des propriétés pharmacologiques des plantes a sans doute été souvent cruciale pour sa survie et cet inventaire est aujourd'hui loin d'être terminé : de l'aspirine issue du saule blanc à des antitumoraux récents comme la vinblastine (issue de la pervenche de Madagascar) ou le taxotère (issu de l'if américain), cette quête s'élargit aujourd'hui aux invertébrés marins. C'est par exemple à partir de l'étude d'une éponge qu'a été élaborée une molécule antivirale comme l'azidothymidine (AZT).

Cependant, il nous semble important d'insister ici sur trois autres aspects de la biodiversité, moins connus mais à notre avis encore plus essentiels pour l'avenir : son rôle comme source d'innovation, sa contribution à des fonctions "non mar-

\footnotetext{
2 Dans le cas des bovins, I'auroch était présent en Europe occidentale mais ne semble pas avoir été domestiqué. Les bovins domestiques auraient été introduits du Proche-Orient (Vigne, 2004).
} 
chandes " et, enfin, son rôle de sentinelle face aux pollutions.

\section{Biodiversité et innovation : la biomimétique}

Observer la nature pour en tirer des innovations utiles n'est pas une idée nouvelle : Léonard de Vinci était un zélateur emblématique de ce principe, qui a sans doute permis à l'homme certaines de ses avancées majeures : aurions-nous rêvé de conquérir les airs sans la contemplation, depuis des millénaires, du merveilleux ballet des oiseaux? Aurions-nous muni nos pieds de palmes pour explorer les océans sans l'observation des oiseaux aquatiques?

Quelques exemples récents vont nous permettre de montrer combien ce principe peut se révéler également fécond pour l'avenir.

Le premier est relatif aux nouveaux matériaux : on connait l'histoire et le succès du velcro, dont les crochets sont imités de ceux de plantes comme la bardane et fournissent un système d'accrochage à la fois rapide, solide et réversible. Un exemple plus récent est celui d'un scarabée du désert de Namibie, Onymacris unguicularis. Dans ce milieu extrême, cet insecte réussit, grâce à la structure très particulière de la surface de ces élytres, à condenser la faible humidité matinale pour en tirer l'eau nécessaire à sa subsistance (Parker et Laurence, 2001). La constitution de tissus reproduisant ces structures est envisagée pour améliorer l'efficacité des filets à brouillard, actuellement utilisés dans des zones de faible pluviométrie, comme la côte pacifique de l'Amérique du Sud, pour fournir de l'eau potable aux villages isolés.

Le deuxième aspect est celui de la locomotion dans divers milieux: ainsi, une analyse hydrodynamique de l'immense nageoire de la baleine à bosse, bordée de gros tubercules irréguliers, a montré que cette morphologie a priori surprenante était beaucoup plus efficace que des structures lisses dans certains types d'écoulements, observation ouvrant des perspectives intéressantes pour des engins comme les voiliers de compétition (Miklosovic et al., 2004). Autre exemple, dans le domaine de la robotique, la création de machines reproduisant la marche bipède des oiseaux ou celle, hexapode, des insectes a montré que ce mode de locomotion pouvait dans certains milieux être plus intéressant que les roues ou la marche bipède ou quadrupède des mam- mifères. Enfin, la fabrication de "nanodrones ", avions sans pilote de la taille d'un gros insecte, amène à tenter de comprendre et reproduire le vol de la libellule, qui peut alternativement être en vol stationnaire ou se déplacer rapidement : on y parvient aujourd'hui à condition que le nanodrone demeure relié à un fil, ce qui montre le chemin restant à parcourir (Constans, 2006).

Nous terminerons ${ }^{3}$ par un aspect qui nous est cher: celui des sociétés de robots et de l'intelligence répartie. En effet, les premiers robots étaient conçus de manière anthropocentrique, c'est-àdire comme des individualités autonomes qui devaient pouvoir capter, traiter et explorer les informations à partir de leurs ressources propres. C'est d'ailleurs l'image que les films de science-fiction voir par exemple R2D2 et Z6PO dans " $L a$ guerre des étoiles" - ont contribué à populariser. Cette conception s'est heurtée à un certain nombre de limites, dès lors que l'on cherchait à augmenter les capacités de ces robots: en particulier, lorsqu'il s'agissait d'évoluer dans un environnement mal connu et hostile (exploration spatiale, installations nucléaires, villes après un séisme, etc.), la taille et le coût de ces robots constituaient souvent un facteur limitant. Or, il existe un autre type d'intelligence - définie ici comme la capacité d'analyser une situation et d'en déduire un comportement approprié que ce modèle "concentré " et individuel qui est le nôtre : c'est celui des insectes sociaux, fourmis, abeilles, termites. Dans ce cas, l'intelligence de chaque individu est relativement restreinte: il ne capte que des signaux locaux et ne peut réaliser qu'un traitement sommaire de ces informations. En revanche, il échange en permanence, à travers des messages chimiques, tactiles ou visuels, les informations qu'il capte avec divers congénères et contribue ainsi à une "intelligence répartie ", qui permet à une colonie de localiser et d'exploiter rapidement une ressource alimentaire, de déplacer son couvain en cas de danger ou de neutraliser un agresseur. C'est sur ce principe que se sont développées les "sociétés de robots", constituées d'un grand nombre de petits robots, peu coûteux et n'ayant que de faibles capacités, mais interconnectés. Une telle configuration présente en parti-

\footnotetext{
${ }^{3}$ Nous invitons le lecteur intéressé par cet immense domaine à interroger un moteur de recherche autour des termes "biomimetics" ou "bionics".
}

culier l'intérêt d'être robuste par rapport aux perturbations, la disparition de quelques unités dans des environnements hostiles ne diminuant pas l'intelligence collective de l'ensemble.

\section{Biodiversité et services écologiques}

Les arguments relatifs à la fourniture d'aliments, de matériaux ou de médicaments ont en commun le fait qu'il s'agit de biens pouvant être mis sur le marché et dont la "valeur" peut donc être estimée par un prix. Or, il existe d'autres fonctions liées à la biodiversité qui ne font pas l'objet d'échanges marchands mais qui n'en sont pas moins importantes: régulation du climat et de la composition de l'atmosphère, fixation des sols permettant de limiter l'érosion, épuration de l'eau, pollinisation des cultures, recyclage de la matière organique, fourniture d'espèces pouvant réguler les ravageurs, etc. Par exemple, l'introduction des bovins en Australie a buté sur l'absence de bousiers capables de traiter les bouses de vache, les espèces locales étant spécialisées dans le traitement des excréments des kangourous. Il a donc fallu importer des bousiers européens pour éviter la stérilisation des pâturages. La valeur du travail de ces bousiers a été estimée à 2 milliards de dollars par an (Anonyme, 2002).

On regroupe ces différentes fonctions sous le terme de "services écologiques" et, depuis une dizaine d'années, des économistes essayent d'estimer par diverses méthodes la valeur de ces services: on peut, par exemple, calculer la perte de productivité des cultures si la pollinisation par les insectes venait à diminuer ${ }^{4}$ ou le coût du traitement des eaux si l'épuration naturelle cessait de fonctionner. Une première estimation globale de l'ensemble de ces services (Constanza et al., 1997) a fourni le chiffre impressionnant de 33000 milliards de dollars par an, soit presque le double du PNB mondial.

Mais ce travail a surtout souligné que la part des biens marchands dans cet ensemble n'était que d'environ 7\%, autrement dit que ces services écologiques gratuits, dont nous n'avons souvent pas conscience, était en fait la partie majeure de l'utilité de la biodiversité.

\footnotetext{
${ }^{4}$ Cette fonction de pollinisation par les insectes a été estimée aux États-Unis à 15 milliards de dollars en l'an 2000 (source : Inra Presse, communiqué du 25 juillet 2005).
} 
Développant cette approche, le Millenium Ecosystem Assessment ${ }^{5}$ a identifié 24 services rendus par les écosystèmes, en distinguant trois catégories :

- les services de prélèvement, qui correspondent à la fourniture - via la productivité des écosystèmes - d'eau, de produits alimentaires, de matériaux, de fibres textiles ou de médicaments ;

- les services de régulation, liés au rôle des écosystèmes dans la réalisation des grands cycles biogéochimiques et à leurs conséquences sur la qualité de l'air, le climat, la limitation de l'érosion et des inondations mais aussi dans la limitation des agents pathogènes et des parasites ou dans la "fourniture " d'insectes pollinisateurs et autres auxiliaries;

- les services culturels, c'est-à-dire qui " alimentent " des valeurs spirituelles, religieuses ou esthétiques, y compris des activités de récréation et de tourisme.

Le Millenium Ecosystem Assessment a, en outre, mis en évidence ce que nous appelons le "paradoxe de la mise en valeur" des écosystèmes: à partir de diverses études de cas, il apparaît que, lorsque l'on aménage un écosystème pour en tirer plus de produits commercialisables (par exemple le développement d'élevage de crevettes dans des zones côtières tropicales ou l'aménagement de forêts pour favoriser la production de bois de qualité), la valeur totale des services rendus diminue de manière souvent considérable. Sans être inéluctable, ce phénomène nous invite à considérer autrement des milieux comme des friches, des jachères, des zones humides, apparemment "improductives " mais impliquées en fait dans la production de services dont la valeur peut se révéler très supérieure à ceux issus des zones "productives".

\section{Biodiversité et vigilance : les bioindicateurs}

Les activités humaines conduisent à libérer dans l'environnement un nombre sans cesse croissant de substances chimiques. Certaines, comme les nitrates et les phos-

\footnotetext{
${ }^{5}$ Cette initiative financée par de nombreux pays, programmes des Nations unies et fondations internationales, a mobilisé de 2001 à 20051360 experts de 95 pays pour mieux évaluer les services rendus par les écosystèmes et leurs contributions au bien-être humain. Elle fait suite aux travaux du Groupe international d'étude du climat (GIEC) qui avait identifié le risque d'une dégradation des écosystèmes suite au changement climatique. Voir le site www.maweb.org.
}

phates, sont, hélas, en concentrations importantes dans de nombreux milieux aquatiques et peuvent être facilement suivies par des méthodes physicochimiques simples et peu coûteuses. Même si des efforts importants sont encore à accomplir, on peut espérer que ces " macropolluants " seront à l'avenir de mieux en mieux contrôlés.

La situation est très différente dans le cas des " micropolluants ", substances présentes à faible concentration mais de plus en plus nombreuses et, en outre, chimiquement très diverses. On cite souvent le chiffre de 100000 molécules différentes produites par l'homme et susceptibles de se retrouver dans l'environnement et 350 molécules nouvelles seraient produites chaque année. Outre cette diversité, leur dosage est souvent coûteux et leurs effets biologiques mal connus. En effet, seules certaines molécules sont soumises à une obligation d'évaluation a priori de leurs effets toxicologiques ${ }^{6}$ et, même dans ce cas, les tests sont réalisés sur un nombre restreint d'espèces et pendant des périodes relativement courtes. En outre, alors que pour des molécules "classiques" on observe une relation assez simple entre les doses et les effets biologiques - augmentation régulière de l'effet en fonction de la dose - qui permet donc de fixer simplement des limites admissibles de concentration, les courbes de réponse des micropolluants sont souvent plus complexes, avec par exemple des relations en "U inversé ", c'est-à-dire des effets biologiques maximaux pour des concentrations faibles ou moyennes ; de ce fait, la définition de doses admissibles à partir de l'extrapolation des effets observés à des doses élevées devient délicate. Enfin, la question complexe des interactions éventuelles entre toutes ces molécules (ou leurs produits de dégradation) et des effets à long terme de ces mélanges ne peut à l'évidence être abordée par ces tests a priori.

C'est pourquoi le suivi de certaines composantes de la biodiversité peut jouer un rôle utile, car permettant une double intégration : l'intégration des effets conjoints

\footnotetext{
${ }^{6}$ C'est tout le débat actuel autour du programme REACH (Registration, Evaluation, Authorisation and Restriction of Chemicals, enregistrement, évaluation, autorisation et restrictions relatifs aux substances chimiques) de I'Union européenne, qui vise à mettre en place un système global d'évaluation et de suivi pour toutes les molécules dont la produc-
} tion serait supérieure à un certain seuil. des différentes substances et l'intégration dans le temps de ces effets, ce que des dosages chimiques ponctuels ne permettent pas. On peut citer comme exemple récent la réduction des populations de bulots de la mer du Nord, du fait d'anomalies de leurs organes génitaux, attribuées finalement aux peintures antisalissures des bateaux, le déclin de la population des alligators de Floride, du fait d'une pollution au DDT causant une réduction de la taille du pénis, ou enfin le développement de poissons intersexués en Grande-Bretagne et en France, sous l'effet de polluants mimant les hormones stéroïdes (Cravedi, 2004).

Même si la perturbation d'une fonction physiologique chez une espèce donnée ne constitue pas forcément une indication d'un risque pour l'homme, ce rôle de "sentinelle" que peut jouer la biodiversité peut à l'avenir se révéler précieux, en fournissant des signaux d'alerte globaux qu'il conviendra ensuite d'analyser plus finement.

\section{Importance du temps long}

Pour conclure sur cette nouvelle vision de l'utilité, nous soulignerons le fait que ces différentes fonctions et services de la biodiversité dont nous pouvons bénéficier aujourd'hui se sont forgés dans la très longue durée. Nous prendrons l'exemple de trois ressources clés de la révolution industrielle qui ont façonné le monde d'aujourd'hui : l'acier, le béton et l'énergie : - l'histoire de l'acier a débuté il y a plus de deux milliards d'années, quand les cyanobactéries présentes dans l'océan ont commencé à libérer de l'oxygène. Celui-ci, avant de se libérer dans l'atmosphère, a d'abord oxydé le fer dissous dans les océans, pour donner les précipitations d'hydroxyde de fer à l'origine des minerais exploités de nos jours. Il faut d'ailleurs souligner que la formation de très nombreux filons métallifères, qui fournissent une ressource concentrée pouvant être exploitée économiquement ${ }^{7}$, implique l'intervention de bactéries. On notera en particulier que nos réserves d'uranium, considérées comme pouvant relayer pour un temps les énergies fossiles, résultent de ce phénomène (Northrop et al., 1990) ;

\footnotetext{
$\overline{7}$ À titre d'exemple, les océans contiennent à l'état dissous $1 \mathrm{mg}$ d'or par mètre cube, soit plus d'un milliard de tonnes d'or au total, à comparer aux 30000 tonnes de réserves de l'ensemble des banques mondiales ; mais cette richesse est économiquement inexploitable !
} 
- les dépôts de calcaire exploité par l'industrie cimentière datent pour la plupart du Jurassique et du Crétacé, c'est-àdire d'une période allant de 200 à 65 millions d'années avant notre ère. L'accumulation lente des minuscules coquilles calcaires d'organismes planctoniques a donc permis l'édification des coquilles humaines d'aujourd'hui que sont nos maisons et autres bâtiments!

- enfin, les dépôts fossiles de pétrole, de gaz et de charbon, notamment ceux de charbon au Carbonifère il y a plus de 300 millions d'années, sont tous issus d'accumulation de matière organique et ont rendu possible la production d'énergie à bas prix qui est à la base du modèle économique des pays développés. Pour souligner la dépendance vis-à-vis de cette activité passée de la biodiversité, on peut calculer que notre planète consomme chaque jour une quantité de charbon équivalente à la production de dix siècles d'énergie fossile !

C'est pourquoi la réflexion sur l'utilité de la biodiversité doit impérativement se placer dans la perspective non seulement des biens et services actuels mais aussi de ceux susceptibles d'être produits à l'avenir et utilisés par les générations futures.

\section{La stabilité : une dynamique permanente}

Le contexte de l'étude de la biodiversité a profondément changé depuis le début du $\mathrm{XVIII}^{\mathrm{e}}$ siècle. Il s'agissait alors d'étudier une situation supposée stable, de visiter l'œuvre du Créateur, et d'en récolter les bienfaits. Ainsi, lorsque Louis XIV envoie en 1700 le botaniste Joseph Pitton de Tournefort en expédition au Levant, ce dernier ne manque pas de gravir le mont Ararat, à la recherche d'éventuels restes de l'Arche de Noé (Laissus, 2003).

La découverte des faunes disparues, puis les hypothèses transformistes du XIX ${ }^{\mathrm{e}}$ siècle, n'ont finalement que peu modifié cette vision statique, dès lors que les espèces apparaissaient, à l'échelle humaine, des entités immuables. La fameuse étude par Cuvier des momies d'ibis des tombes de Thèbes, en tous points semblables aux ibis actuels, même si elle ne constitue pas, comme le souhaitait Cuvier, un argument contre le transformisme, illustre la persistance de ce point de vue (Laissus, 2003). En outre, le fait d'attribuer la disparition des faunes passées à des événements cataclysmiques (déluges, météorites géantes...) pouvait conforter l'idée qu'entre ces événements rarissimes, les écosystèmes étaient des états d'équilibre stable au sens dynamique du terme, c'est-à-dire pouvant revenir à cet état d'équilibre après des perturbations éventuelles.

L'un des grands changements conceptuels de la fin du $\mathrm{Xx}^{\mathrm{e}}$ siècle a été à notre avis l'abandon - ou du moins la relativisation - de cette notion d'état d'équilibre en biologie et en écologie. Cette évolution résulte à la fois d'observations empiriques et de progrès conceptuels.

\section{Les écosystèmes ont une histoire}

Sur le plan empirique, l'observation fine des écosystèmes et de leur histoire a conduit à mettre en évidence le caractère souvent contingent de leur état à un instant donné, résultant de phénomènes historiques (arrivées ou disparitions d'espèces dans les biocénoses, "catastrophes " plus ou moins périodiques, impacts durables des environnements passés, etc.). Ainsi, le faible nombre d'espèces de poissons dans les eaux douces des pays d'Europe du Nord (une quarantaine en moyenne), qui contraste avec la grande diversité des pays méditerranéens (par exemple près de 100 espèces en Grèce et plus de 150 espèces en Turquie), s'explique essentiellement par le rôle de refuge qu'a joué le sud de l'Europe lors des dernières glaciations et reflète donc une dynamique de recolonisation qui est peut-être encore en cours. De même, la structuration complexe actuelle des peuplements de chênes blancs européens, ensemble comprenant huit espèces, ne peut se comprendre qu'en retraçant les routes de migration depuis la fin de la dernière glaciation, mais aussi les importantes interventions humaines sur ces peuplements (Petit et al., 2002).

Cette vitesse de transformation des écosystèmes est d'ailleurs impressionnante : on peut calculer que la remontée des chênes vers le nord de l'Europe au Néolithique s'est faite au rythme de plusieurs centaines de mètres par an (Kremer et Petit, 2001). Une telle modification des écosystèmes sous l'effet de facteurs environnementaux peut s'observer aujourd'hui sur des pas de temps extrêmement courts: en une trentaine d'années, l'enrichissement progressif du golfe de Gascogne en espèces subtropicales et la raréfaction des espèces d'eau froide ont été spectaculaires (Blanchard et Vandermeirsch, 2005). Des changements de l'aire de répartition de plus d'un kilomètre par an sont fréquemment enregistrés : de 1975 à 2004, la limite nord de répartition des oiseaux présents l'hiver en Amérique du Nord s'est décalée en moyenne de 1,5 kilomètre par an (La Sorte et Thompson, 2007) ; dans le Bassin parisien, la chenille processionnaire du pin a migré également de 2,7 kilomètres par an pendant cette période (Battisti et al., 2005).

De ce fait, un état observé d'un écosystème, même considéré comme " naturel ", ne peut être considéré sans prudence comme un "état de référence" qu'il convient de préserver ou, en cas de perturbations humaines ou naturelles, de "restaurer".

Toujours sur un plan empirique, de nombreuses opérations de protection ou de restauration visant en particulier à diminuer l'impact anthropique ont montré leurs limites, avec notamment la disparition d'espèces inféodées, souvent de manière complexe, aux activités humaines. Le narcisse des Glénans est un exemple emblématique de cette difficulté. Cette espèce endémique était menacée par la surexploitation (arrachage des bulbes) et a été classée parmi les espèces protégées par la Convention de Berne de 1979. Mais il est apparu que sa persistance n'était possible que si la fauche ou le pâturage des moutons maintenaient une prairie rase, d'où la nécessité d'encourager ces pratiques. En outre, le développement excessif des goélands, eux-mêmes protégés, conduit à un enrichissement du sol en azote qui semble préjudiciable à cette plante (Guy, 1990). On peut d'ailleurs étendre cette observation à de nombreuses plantes prairiales (orchidées, flore alpine), qui dépendent fortement du maintien d'une pelouse pâturée.

\section{L'équilibre stable : une singularité théorique}

Sur un plan conceptuel, les progrès de la modélisation et la possibilité de suivre le comportement pas à pas d'un système au lieu d'être seulement à même de calculer son éventuel état asymptotique - ont montré combien des équilibres stables ne constituaient que des cas très particuliers d'évolution de systèmes régis par des lois et des paramètres constants : des modèles de comportements fluctuants plus ou 
moins périodiques, voire chaotiques, ont été développés par la dynamique des populations et des peuplements (Costantino et al., 1997 ; Ricard, 2003). Ils ont montré en particulier qu'il n'était pas nécessaire de rechercher systématiquement l'influence forte d'un facteur environnemental pour expliquer une évolution majeure d'un écosystème. En effet, des phénomènes non linéaires (effets de seuils, points critiques, etc.) peuvent conduire à deux évolutions très différentes pour deux systèmes initialement similaires. Popularisée par René Thom avec la notion mathématique de "catastrophe" (Thom, 1989), cette nouvelle vision s'est peu à peu étendue à de nombreux domaines des sciences, en particulier de la biologie.

De manière moins radicale, ces approches ont permis également de montrer que le temps nécessaire pour atteindre un nouvel équilibre stable était souvent très supérieur à la durée de stabilité des paramètres biologiques ou écologiques déterminants. De ce fait, l'état d'équilibre, même s'il existe, apparaît comme une abstraction sans grande pertinence concrète.

La manière de considérer les perturbations a elle-même changé, dès lors qu'il est apparu que des événements comme les incendies, les inondations, les tornades, étaient nécessaires à la régénération ou à la dissémination de certaines espèces, qui disparaissaient donc dans des milieux trop "protégés ". Cette hypothèse de la "perturbation intermédiaire ", c'està-dire du rôle positif d'un régime de perturbations modérées sur la biodiversité, a été émise par Connel (1978) à propos des massifs coralliens et théorisée notamment par Huston (1979). Elle a été testée dans des situations variées, par exemple pour expliquer les variations de biodiversité de la végétation rivulaire des fleuves en fonction du régime hydraulique (Tabacchi et al., 1990 ; Bendix, 1997 manque référence Bendix, 1997). La décision de ne plus lutter contre les incendies dans les parcs nationaux américains, sauf en cas de danger pour les populations, illustre bien cette nouvelle perception du rôle parfois bénéfique des perturbations ${ }^{8}$. Certains ont même proposé de s'inspirer du mode d'action des incendies pour développer des pratiques durables d'exploitation des forêts : c'est le concept de "sylviculture simulée" (McRae et al.,

\footnotetext{
${ }^{8}$ On se gardera bien sûr de généraliser cette observation à l'ensemble des perturbations, en particulier celles dues aux activités humaines.
}

2001), qui constitue donc un autre exemple de biomimétisme !

Cet intérêt pour les perturbations temporelles s'est accompagné d'une prise en compte croissante de l'hétérogénéité spatiale. Comme nous l'avons vu, le passage du concept d'écosystème, considéré comme homogène en tout point, à celui de paysage, constitué de multiples discontinuités susceptibles de moduler la structure de la biodiversité, est une évolution forte et assez récente de la pensée écologique. Se dégage donc peu à peu une vision selon laquelle l'hétérogénéité spatiotemporelle d'un milieu serait le moteur d'une dynamique permanente, elle-même nécessaire au maintien et à l'évolution de la biodiversité. Certains théoriciens (O'Neill, 2001) considèrent même qu'il y a une contradiction logique entre les notions d'homogénéité et de stabilité d'un écosystème et que l'hétérogénéité spatio-temporelle de l'écosystème est une condition nécessaire à une certaine stabilité de ses propriétés fonctionnelles.

Cette nouvelle vision rompt avec une longue tradition de l'écologie: la notion de climax, terme désignant la structure d'équilibre - en termes de composition et d'abondance relative des espèces - d'une communauté végétale a été un concept majeur tout au long du $\mathrm{xx}^{\mathrm{e}}$ siècle. Confronté à l'observation de perturbations naturelles récurrentes des communautés au niveau local empêchant d'atteindre cet état d'équilibre, certains théoriciens ont réintroduit la notion d'équilibre au niveau du "paysage " (ensemble des unités écologiques interconnectées d'une région) en dénommant métaclimax l'équilibre dynamique entre ce paysage et un régime de perturbation supposé stable à cette échelle. La longue histoire de cette idéologie de "l'équilibre de la nature", qui a conditionné une conception "fixiste" de sa conservation, est décrite en particulier par Blandin (2004).

Cette nouvelle perspective amène donc à considérer la situation instantanée d'une population, d'une espèce ou d'un écosystème non pas comme un état stable et optimum mais comme un point sur une trajectoire, qui peut n'être elle-même que l'une des trajectoires possibles. C'est d'ailleurs sans doute cette dynamique qui confère aux écosystèmes leurs propriétés de résistance (capacité à conserver leurs propriétés fonctionnelles en cas de perturbations modérées) et de résilience (capacité à retrouver ces propriétés après des perturbations plus fortes). De ce fait, la notion de gestion durable prend une connotation très différente de celle relative aux ressources non renouvelables (pétrole, minerais), pour lesquelles elle implique essentiellement une gestion économe permettant aux générations futures d'accéder à ces ressources. Dans le cas de la biodiversité, la question devient celle de "l'adaptabilité durable", à savoir l'identification des composantes ou des processus d'un système écologique qu'il convient de préserver, renforcer, modifier pour que son évolution ne handicape pas les générations futures, voire leur bénéficie. Comme l'écrivaient dès 1984 Lamotte et al. (voir également Blandin, 1986) : "Conserver la nature, c'est lui conserver ses potentialités évolutives." (Lamotte et al., 1984).

\section{Des conséquences tant théoriques que pratiques}

Un tel énoncé permet de mesurer l'ampleur du défi posé à la science : abandonner le paradigme de "l'équilibre harmonieux des écosystèmes ", c'est accepter de soumettre à un examen critique l'état d'un écosystème, c'est-à-dire reconnaître la possible légitimité d'un certain nombre de pratiques (introduction ou éradication d'espèces, modification des habitats...) jugées jusqu'alors regrettables, car "modifiant l'équilibre " ou ne visant pas à revenir à un état de référence antérieur. Mais en même temps, il importe de reconnaître que ce nouveau paradigme - "Tout est perturbation " n'est pas non plus exempt de connotation idéologique et peut conduire, à l'extrême, à une déconstruction de la nature nous dédouanant de toute éthique environnementale.

Ainsi, Génot (2006) affirme la nécessité "politique" de continuer à mettre en avant ce concept d'état d'équilibre, même s'il est scientifiquement contestable, pour éviter de telles dérives : "À force de glorifier le changement et de mettre sur le même plan perturbation naturelle et anthropique, on soutient l'idée qu'il n'y a pas de lois de la nature à respecter, donc pas d'éthique à adopter vis-à-vis de la nature. Les scientifiques veulent gérer des écosystèmes tout en rejetant toute notion d'état de référence. (...) Si communauté et équilibre posent un problème sur le plan scientifique, ils doivent être mis en avant pour une éthique guidant les rapports entre l'homme et la nature, sous peine de voir Homo destructor ne jamais devenir Homo ecologicus. " 
De manière plus fondamentale, Blandin et Bergandi (1997) soulignent que cette prise en compte de la dimension historique et contingente des écosystèmes peut être redoutée, et donc refusée, par certains chercheurs travaillant sur la biodiversité, car susceptible d'affaiblir le statut scientifique et épistémologique de leurs études : la description, même détaillée, d'événements uniques, non reproductibles, est souvent considérée comme ne permettant guère la découverte de lois de portée générale et la "scientificité" des disciplines se livrant à de telles études est, selon la pyramide classique - mais toujours prégnante dans certains esprits d'Auguste Comte, considérée comme inférieure.

\section{Conclusion :} de l'exploitation

\section{des ressources}

\section{à la gestion des services}

L'évolution, que nous venons de décrire, de nos conceptions de l'utilité et de la stabilité de la biodiversité modifie assez profondément la manière de concevoir l'utilisation des "ressources " naturelles vivantes ou issues du vivant.

Dans une conception classique, on puisait dans la nature des ressources - produits de la pêche, produits forestiers, productions agricoles - qui, parce qu'elles étaient produites par des êtres vivants, étaient qualifiées de "renouvelables". Face à la perception du caractère non inépuisable de ces ressources se sont mis en place divers systèmes de régulation quotas, réserves, périodes d'exploitation -, mais qui restaient tous centrés sur la ressource et son exploitation marchande optimale et visaient à "équilibrer "les prélèvements et le renouvellement de la ressource, à l'image des modèles de dynamique des populations utilisés en halieutique et en foresterie.

Ces modèles ne prenaient donc pas en compte les effets de cette exploitation sur d'autres services des écosystèmes - la forêt étant par exemple considérée uniquement dans sa fonction de production de bois - ni même les effets indirects de cette exploitation, au-delà de l'effet direct des prélèvements, sur l'évolution de la ressource elle-même. Des crises emblé- matiques, comme l'effondrement des stocks de morue de Terre-Neuve, des remises en cause par la société de gestion trop "mono-usage" de certains milieux, comme les forêts domaniales périurbaines, ou la mise en évidence de la dégradation de certains services non marchands, comme la qualité des eaux, ont montré les limites de ces modes d'exploitation classiques.

On est donc amené à passer de cette vision d'exploitation équilibrée d'une ressource à celle d'une gestion dynamique d'un écosystème et de l'ensemble des biens et services qu'il produit, avec, comme nous l'avons indiqué, la préoccupation de la fourniture à long terme de ces biens et services. Cette ambition de gestion durable suppose l'élaboration de nouveaux savoir-faire, qui devront combiner à la fois les compétences des sciences écologiques et des sciences humaines pour définir des stratégies adaptées. En effet, il apparaît illusoire de vouloir gérer cette question de manière technocratique, par exemple en définissant une "valeur totale " des services d'un écosystème. Les choix qui seront faits seront donc des compromis, des ajustements permanents entre des attentes sociales, elles-mêmes multiples, et les capacités des écosystèmes à y répondre à court terme tout en assurant leur viabilité à long terme.

\section{Références}

Anonyme. Les coprophages et la dégradation des excréments. Revue GARDE 2002 ; 46 : 2-6.

Battisti A, Stasny M, Netherer S, et al. Expansion of geographic range in the pine proces sionary moth caused by increased winter temperatures. Ecol App/ 2005 ; 15 : 2084-96.

Blanchard F, Vandermeirsch F. Warming and exponential abundance increase of the subtropical fish Capros aper in the Bay of Biscay (1973-2002). C R Biol 2005 ; 328 : 505-9.

Blandin P. Bioindicateurs et diagnostic des systèmes écologiques. Bulletin d'écologie $1986 ; 17: 215-307$.

Blandin P, Bergandi D. Entre la tentation du réductionisme et le risqué d'évanescence dans l'interdisciplinarité : I'écologie à la recherche d'un nouveau paradigme. In : La crise environ nementale. Les Colloques, $\mathrm{n}^{\circ} 80$. Paris: Inra éditions, 1997.

Chevassus-au-Louis B, Barbault R, Blandin P. Que décider? Comment? Vers une stratégie nationale de recherche sur la biodiversité pour un développement durable In: Chevassus-auLouis B, Barbault R, eds. Biodiversité et change ments globaux. Enjeux de société et défis pour la recherche. Paris : éditions ADPF ; ministère des Affaires étrangères, 2004. 'www.adpf. asso.fr/adpf-publi/folio/textes/biodiversite.pdf.

Chevassus-au-Louis B. La biodiversité : un nouveau regard sur la diversité du vivant. I. Immensité et complexité. Cah Agric 2007 ; 16 219-27.
Connel JH. Diversity in tropical rain forests and coral reefs. Science 1978 ; 199 : 1302-10.

Constans N. L'essor des robots insectes. Recherche 2006 ; 394 : 67-73.

Costantino RF, Desharnais RA, Cushing JM, Dennis B. Chaotic dynamics in an insect population. Science $1997 ; 275$ : 389-91.

Costanza R, d'Arge R, de Groot R, et al. The value of the world's ecosystem services and natural capital. Nature $1997 ; 387$ : 253-60.

Cravedi JP. Alimentation et environnement : le cas des perturbateurs endocriniens. Conférence au forum "Aux bons soins de l'alimentation ", Toulouse, 14 décembre 2004. [disponible sur www.agrobiosciences.org].

Génot JC. Vers un changement climacique? Courrier de l'Environnement de I'INRA 2006 ; $53: 129-32$.

Guy P. De I'avenir de la diversité biologique chez les végétaux. Courrier de l'Environnement de I'INRA $1990 ; 12: 15-24$ [Pour des précisions sur le narcisse des Glénans, voir: cisions sur le narcisse des
http ://ccrijsp.mnhn.fr/cbnbp].

Huston M. A General Hypothesis of Species Diversity. The American Naturalist 1979; 113 : 81-101.

Kremer A, Petit R. L'épopée des chênes européens. Courrier de l'environnement de I'INRA $2001 ; 21$ : 133-8.

La Sorte FA, Thompson FR. Poleward shifts in winter ranges of North American Birds. ECO $\log 2007 ; 88$ : 1803-12.

Laissus Y. Le Muséum national d'histoire naturelle. Ed. Découvertes. $2^{\mathrm{e}}$ éd. Paris : Gallimard 2003.

Lamotte M, Sacchi CF, Blandin PArticle "Écologie ". Encyclopaedia Universalis. $2^{\mathrm{e}}$ édition. 1984 ; vol 5

McRae DJ, Duchesne LC, Freedman B, Lynham TJ, Woodley S. Comparisons between wildfire and forest harvesting and their implications in forest management. Environ Rev $2001 ; 9$ : 223-60.

Miklosovic DS, Murray MM, Howle LE, Fish FE Leading-edge tubercles delay stall on humpback whale (Magaptera novaengliae) flippers. Phys Fluids 2004; 16 : L39-L42.

Northrop HR, Goldhaber AB, Landis GP Unruh JW. Genesis of the tabular-type vanadium-uranium deposits of the Henri Basin, Utah. Econ Geol 1990 ; 85 : 215-36.

O'Neill V. Is it time to bury the ecosystem concept? Ecology $2001 ; 82$ : 3275-84.

Parker AR, Laurence CR. Water capture by a desert beetle. Nature 2001 ; 414 : 33-4.

Petit RJ, Csaikl M, Bordacs S, et al. Chloroplast DNA variation in European white oaks. Phylogeography and patterns of diversity based on data from over 2600 populations. For Ecol Manage 2002 ; 156 : 5-26.

Porcher J. L'esprit du don: archaïsme ou modernité de I'élevage? Rev MAUSS 2002 $20: 245-62$.

Ricard J. La complexité biologique. Pour la Science $2003 ; 314$ : 30-3.

Tabacchi E, Planty-Tabacchi AM, Décamps O. Continuity and discontinuity of the riparian vegetation along a fluvial corridor. Landscape Ecology $1990 ; 5: 9-20$.

Thom R. Paraboles et catastrophes. Paris: Flammarion, 1989.

Vigne JD. Les débuts de l'élevage. Collection "Le collège de la Cité ". Paris: éditions Le Pommier-La Cité, 2004. 